\title{
Syllable Structure for an Artificial Language Based on Universal Principles
}

\author{
Stuart Davis \\ Indiana University
}

\begin{abstract}
This paper discusses the types of syllables that should b e f o undin a spoken artificial language based on the common types of syllables found in naturallanguages. The paper also examinesthevarious units within the syllable, such as onset, nucleus, and coda, suggesting how the preferred structure of these units may be incorporated into an artificial language. One proposal that will emerge is that an artificial language needs to allow for at least a slight degree of complexity in its syllable structure. The paper further discusses related issue of prosody such as word stress and minimal word length in an artificial language. The paperconcludeswith an overview oftheproposals made about syllable structure for an artificial language.
\end{abstract}

\section{Basic Syllable Structure and Syllable Types}

Natural languages display a wide variety of syllable types. These 
can include very simple syllables where a consonant is followed by a vowel (that is, the CV syllable) as in the English word "papa" consisting of two CV syllables. They also can include very complex syllables as illustrated by the English word "sprints" which has three consonants before the vowel and three consonants after the vowel, but is nonetheless perceived as a single syllable by English speakers. Moreover, the syllable in natural language can be divided into three subunits or constituents: onset, nucleus, and coda. The onset is a unit consisting of the syllable-initial consonant or consonants. While the onset in the syllable "pa" issimple, consisting of just the phoneme /p/, the onset in "sprints" is complex, consisting of "spr". The nucleus is the peak of the syllable; it is the loudest part of the syllable, and it is the only unit of the syllable that is always obligatory. It typically consists of a vowel (or diphthong) but in some languages may be a syllabic consonant as well. Ifoneconsidersthe syllable "pa", the vowel /a/ comprises the nucleus. Notice that we would still have a complete syllable without the onset; it would just be [a]. Consequently, it is possible to have an onsetless syllable.However, if wedidnothavethe nucleus, the syllable would be incomplete; thus [p] without any vowel is an impossible syllable. Finally, the coda is a unit consisting of the final consonant or consonants of the syllable. Like the onset, it is not an obligatory unit. Thus the syllable "pa" has no coda. The English syllable "sprints" has three consonants in its coda, while the syllable "top" has a single coda consonant. Below the syllable and its units are shown.

$\mathrm{O}=$ onset, $\mathrm{N}=$ nucleus, $\mathrm{C}=$ coda

$$
\begin{array}{ccc}
\multicolumn{3}{c}{\text { Syllable }} \\
/ & \mid & \text { I } \\
\text { O } & \mathrm{N} & \mathrm{C}
\end{array}
$$

While there are other views of syllable-internal structure (see Blevins 1995), the flat structure shown above is argued for at length 
by Davis (1988) and, moreover, such a structure is tacitly assumed in current phonological work within the framework of optimality theory (e.g., Prince \& Smolensky 1993, Kager 1999).

Given the flat syllable structure above, a simple syllable is one that consists of zero or one onset consonant followed by a short vowel, which is $(\mathrm{C}) \mathrm{V}$. Anything larger is a complex syllable. Thus a complex syllable might contain a coda, for example, a CVC syllable; it might contain a diphthong or long vowel, for example [boI], a CVV syllable; it might contain a complex onset, for example "spa" or any combination of these as in the syllable "sprints" which has both a complex onset and a complex coda.

The simple CV syllable is the only syllable type that occurs in all languages. A few languages such as Hawaiian allow only simple syllables. Hawaiian allows both types of simple syllables, CV and V. There are never any coda consonants or complex onsets. A vowel sequence such as CVV in Hawaiian is usually considered as a sequence of two syllables, $\mathrm{CV}$ and $\mathrm{V}$, rather than as a single syllable with a complex nucleus. Many languages such as Arabic do not allow vowel initial syllables at all. In most languages that do allow for complex syllables, the complexity is minimal. For example, Korean syllables may have a coda consonant, but onsets and codas typically can only consist of a single consonant, so that these subunits themselves are never complex. Arabic is similar to Korean in this respect, though in Arabic a complex coda can occur at the end of a word. Spanish tends not to have complex codas but can have a complex onset consisting of two consonants. English allows for much more complexity in its syllables, as seen by the example "sprints" where the onset and the coda each consists of three consonants. Such complexity is actually quite rare in the world's languages. Given the variety of syllable types that can occur in the world's languages, the question that emerges concerns what should the syllable types be in an artificial language. This is the question that will be discussed in the following section. 


\section{Syllable Types in an Artificial Language}

As mentioned above, the simple CV syllable is the only syllable type found in all natural languages. What is the implication of this for an artificial language? One temptation would be to construct an artificial language, so that all the syllables in the language are CV with no variation in the syllable types. However, there are certain problems in requiring all syllables to be $\mathrm{CV}$. One problem is that assuming a relatively simple phonemic inventory is maintained for the artificial language, requiring all syllables to be $\mathrm{CV}$ would have the consequence offorcing manyofthewordsintheartificiallanguagetobequitelong. In order to express all the vocabulary items, there would be many words that would be CVCVCV with three syllables or CVCVCVCV with four syllables. It would seem that vocabulary would be easier for the learner to remember if the items were short. Natural languages that do have only simple syllables compensate for it in two ways. One way which is illustrated by Hawaiian is to allow for words to be long and to have vowel initial syllables. This expands the number of potential permissible words in the language. The other way is for a language to be a tone language. A tone language, such as Chinese or many of the African languages, allows for phonemic distinctions to be made by the pitch of the voice. Thus a CV word such as "ma" may have one meaning if said with a high pitch but a completely different meaning if said with a different pitch. Consequently, many tone languages such as Mandarin Chinese and the African language Yoruba have mostly simple syllables since tone is used phonemically.

With respect to an artificial language, neither of the above strategies for keeping syllables simple seems to be practical. Making words quite long in order to maintain CV syllable structure would make the vocabularydifficult to learn.Moreover, having tonal distinctions like in Chinese for an artificial language would make the language extremely difficult to learn for the many people who do not speak a tone 
language. Consequently, an artificial language must require some complexity in its syllable types. The main question then becomes what sorts of complexities should be allowed. This is the question addressed in the following sections. We will consider this question by specifically examining each of the subunits of the syllable.

\section{The Syllable Onset in an Artificial Language}

As discussed above, it is impractical to require an artificial language to maintain only CV syllables. Somecomplexity must be allowed. One possibility is to allow for complex onsets. If one examines complex onsets in natural languages, there are certain types of complex onsets that are preferred. The main type thatis preferred is one where the first consonant in the cluster is an obstruent, typically a stop consonant, and the second one is a sonorant, typically a liquid. This illustrated by the English examples "play" and "cry". While English allows for more complex onsets than just these, some languages such as Spanish allow only complex onsets that consist of obstruents followed by liquids and no others. Oneproblem, however, with complex onsets is that they are difficult to pronounce for speakers of languages that do not have them. For example, Cairene Arabic does not have any complex onsets. Usually when Cairene Arabic speakers learn English they insert a vowel between the consonants in words like "play" and "cry", thus pronouncing them as two syllables. Given this, one could ask if any complex onsets at all should be allowed in an artificial language? We suggest that there is one type of onset cluster that is typologically quite common that could be included in an artificial language. This is the cluster $[\mathrm{kw}]$ as in the English word "queen". Thisclusteris not always recognized as an onset cluster since the [w] is often viewed as being part of a complex nucleus, but the cluster is common enough occurring in such languages as Korean and Spanish. Thus, if the syllable types of an artificial language were to be expanded so as to include a 
complex onset, the expansion should probably first include [kw].

In this regard, it is interesting to compare the suggestion here with what is found in the artificial language Esperanto. Because the vocabulary of Esperanto draws heavily from Latin and other European languages, no care seems to have been taken to avoid incorporating words with complex onsets. For example, one finds in Esperanto (Cresswell \& Jartley 1968) such words as "strang" meaning stranger and"knab"meaning boy. The formerword has a three consonant onset, while the latter has the unusual onset cluster $/ \mathrm{kn} /$. The pronunciation of such words would be quite difficult forspeakers of many languages. The latter is particularly difficult for native speakers of English.

A further question regarding the onset of an artificial language is whether or not every consonant in an artificial language should be allowed in the onset. If one examines natural languages, there appear to be two consonants that are sometimes avoided in onset position, though this is not typically discussed in detail. The two consonants are the velar nasal and the liquid consonants $/ 1 /$ or $/ \mathrm{r} /$. It is interesting that Korean tries to avoid placing velar nasals and liquids in onset position. English avoids placing the velar nasal in onset position while the Turkic language Yakut avoids /r/ in onset position. It is possible that in an artificial language, these consonants should also not appear in the onset. This is especially true for the velar nasal, where, typologically it is quite common for it to only appear in the coda.

\section{The Nucleus in an Artificial Language}

Anotherwaytoexpandthesyllable types beyond CV in an artificial language is to allow for a complex nucleus. The main way that natural languages show a complex nucleus is by allowing for long vowels or diphthongs in the nucleus, that is, by having CVV syllables. Given this choice, it is probably preferable for an artificial language to allow diphthongs in the nucleus as opposed to long vowels. This is because 
most languages have diphthongs, but many languages do not have long vowels.Moreover, languages that had long vowels at one stage in their history have lost them at a later stage. This appears to be what is happening in modern Korean (Seoul dialect) where dictionaries indicate vowel length distinctions (such as /pa:m/ "chestnut" vs. /pam/ "night") which are no longer maintained by younger speakers. Similarly, the historical length distinctions in English vowels are perceived as quality differences rather than length differences by American English speakers. This suggests that it may not be viable for an artificial language to have a length distinction. In this regard it is interesting to note that Esperanto does not maintain a vowel length distinction.

On the other hand, expanding the $\mathrm{CV}$ syllable type to $\mathrm{CVV}$ by allowing for a diphthong may be easier to maintain. Diphthongs like /ay/ and /aw/ as in the English words "by" and "cow" are not uncommon and usually do not present problems for the learner. They could be incorporated into an artificial language. And, in fact, both these diphthongs appear in Esperanto. If only one diphthong were allowed, it would probably be preferable just to have /ay/ and not /aw/. This is because the off-glide part of the diphthong in /aw/ is w-like, as in the word "queen" where it is an on-glide part of the onset. By having only the diphthong /ay/ and only the complex onset $/ \mathrm{kw} /$, a certain clarity would be kept in the language: a /w/ on-glide is considered part of the onset, there is no /w/ off-glide; a /y/ off-glide as in "by" /bay/ is part of the nucleus, there is no /y/ on-glide. This fits well with the treatment of these sounds in languages like English and Korean where $/ \mathrm{y} /$ is more vocalic and $/ \mathrm{w} /$ is more consonantal (Davis \& Hammond 1995).

\section{The Coda in an Artificial Language}

There is a third way to expand syllable types beyond CV. This is 
to allow for syllables to have a single coda consonant, that is, to permit CVC syllables as well as CV. Allowing for a single coda consonant is typologically common in natural languages.Languages such as Korean, Mandarin Chinese, and Japanese all allow for CVC syllables. An interesting question arises as to whether the single coda consonant should be restricted in any way. A comparison between the three languages just mentioned reveals interesting differences as to what types of consonants are permitted in the coda. In Korean, both obstruent consonants and sonorant consonants can occur in coda position. Specifically, in Korean, syllables can end in the sounds [p], $[\mathrm{t}],[\mathrm{k}],[\mathrm{l}],[\mathrm{m}],[\mathrm{n}]$, and [?]. But syllables cannot end in aspirated sounds or fricatives such as [s]. Coda consonants in Mandarin Chinese and Japanese are even more restricted. For example, in Mandarin Chinese, syllables can only end in sonorant consonants, specifically nasal consonants or $[\mathrm{r}]$. Obstruent consonants are not permitted in coda position. Japanese witnesses a somewhat different restriction on coda consonants. In Japanese, a coda consonant must either be a nasal or an obstruent that is the first part of a geminate (or long) consonant. Consider, for example, the Japanese word "Nippon" which can be transcribed as [nip.pon]where the period indicates the division between the two syllables. The coda consonant of the second syllable is the nasal [n]. The coda consonant of the first syllable is [p]; however, the [p] here is the first part of a geminate which comprises both the coda of the first syllable and the onset of the second. This is the only situation where an obstruent in Japanese can be a coda, when it is part of a geminate consonant; a short obstruent cannot comprise a coda.

Restrictions like those in Korean, Mandarin Chinese, and Japanese are quite common in natural languages. Two other similar examples come from Italian and the Polynesian language Trukese. In Italian, a coda consonant can be any sonorant, the fricative [s], and any other obstruent as long as it is the first part of a geminate. Trukese makes a further distinction between a coda at the end of the word and a coda elsewhere in the word. A word-final coda can be any single consonant 
while elsewhere in the word a coda consonant can be any consonant as long as it is the first part of a geminate. Other languages, such as English and Arabic, have virtually no restrictions on the nature of a coda consonant. Standard Arabic has none while English has a minor restriction that disallows $/ \mathrm{h} /$ from surfacing in coda position. Moreover, a language such as Hawaiian discussed earlier which allows syllables only to consist of $\mathrm{CV}$ or $\mathrm{V}$ has a complete prohibition on coda consonants.

Given the range of possible coda types and their restrictions that occur in natural languages, it can be asked what might be the preferred coda type for an artificial language. One common type of consonant that seems to be permitted in at least some form in most, if not all, languages that allow for a coda consonant is the nasal consonant. All of the languages discussed above, except Hawaiian which has no coda consonants, permit nasal consonants in coda position. Thus, if an artificial language were to permit complex syllables with coda consonants, the simplest way to do this would be to restrict the coda consonant to a nasal. Having other consonant types in the coda could make learning the pronunciation difficult depending on one's native language. By contrast, in Esperanto any single consonant can appear in the syllable coda as well as typologically unusual consonant clusters such as [md] as in the word "fremd" meaning strange.

As a final question regarding consonants, if an artificial language allows for a nasal consonant to appear in coda position, should there be any restrictions on what that nasal consonant should be? From the perspective of natural languages, $/ \mathrm{m} /$ and $/ \mathrm{n} /$ are by far the two most common nasals. We would suggest that only these two consonants be allowed in coda position. A third nasal consonant that is often found in natural language is the velar nasal /?/. However, the velar nasal frequently has limited distribution in the languages in which it occurs. For example, in Korean and in English, the velar nasal can only appear as a coda consonant; but, on the contrary, in various African languages the velar nasal only appears in onset position. Consequently, it may be 
best to restrict the coda position in an artificiallanguage to $/ \mathrm{m} /$ and $/ \mathrm{n} /$.

\section{Other Issues of Prosody}

Besides syllable structure, there are a number of other issues of prosody that one can considerforanartificiallanguage. Two issues to be considered here are word stress and minimal word length. The issue of stress will be addressed first followed by a discussion of minimal word length.

It was mentioned earlier that an artificial language probably should not be a tone language, given the difficulty of mastering tonal contrasts by those whose native language is not a tone language. This suggests that an artificial language should be a stress language instead. In a stress language, one syllable is more prominent than another. In terms of pronunciation, this means that the stress syllable is pronounced slightly louder and with more emphasis than the other syllables of the word. The question that arises for an artificial language is which syllable of the word should receive the stress. In terms of natural language, stress patterns have been well-studied. Three of the most important works in this regard are Hyman (1977), Halle \& Vergnaud (1987), and Hayes (1995). These works show that the two most common patterns of word stress among languages are languages that have stress on the initial syllable of the word and languages that have stress on the final syllable of the word. Another important factor for some language in determining the location of stress in a word is the presence of a complex nucleus or a coda consonant. In many, though not all languages that have a complex nucleus, a syllable with a complex nucleus attracts stress. Moreover, in some of the languages that allow for a coda consonant, a syllable with a coda consonant may attract stress in the word. These two syllable types (e.g., syllables with a complex nucleus and syllables with a coda consonant) are referred to as heavy syllables in the literature on stress. One interesting difference 
between these two types of heavy syllables is that a syllable with a complex nucleus is much more likely to attract stress than a syllable with a coda consonant. Given this, what might be a simple system of word stress in an artificial language, that allows for a limited number of heavy syllable types? The simplest system always has stress on the same syllable for every word. It is suggested here that for an artificial language, word stress should always be on the initial syllable. Further, in order to keep to this principle of initial stress, it is suggested that syllables with a complex nucleus be restricted to the initial syllable of the word. This restriction would help to avoid whatwould be an almost certain tendency to stress a non-initial syllable with a complex nucleus. However, in this regard it is interesting to note that while Esperanto has consistent stress on the penultimate (next-to-last) syllable, diphthongs (e.g., complex nucleus) can occur in other positions as in words like "apenau" meaning barely which has a diphthong in the final syllable. There would be a tendency to stress wrongly the final syllable in this word rather than the penultimate one. The proposal in this section restricting a complex nucleus (e.g., a diphthong) to the stressed syllable avoids the difficulty encountered in Esperanto.

Regarding syllables that end in a nasal, it is suggested that they not be restricted just to the first syllable. They could appear as a second syllable or even third syllable, without stress. Since studies on stress show that there is less of a tendency to stress syllables with a coda consonant (as opposed to ones with a complex nucleus), it seems quite plausible to permit non-initial syllables with a nasal consonant in the coda without there being a tendency forsuch syllables to attract stress.

As a final point regarding word prosody, researchers such as McCarthy \& Prince (1995) have observed that many languages have minimal word restrictions requiring words to consist of one heavy syllable or two syllables. That is, in many languages a word consisting of a simple CV syllable is prohibited.However,this restriction may not be necessary for an artificial language. First, there are quite a few languages, such as Korean, that do not observe the restriction. In 
addition, second, children in acquiring their first language often start out with words consisting of a single CV syllable. This suggests that while the appearance of a minimal word restriction in many natural languages is interesting, there is no need to incorporate it in an artificial language.

\section{Summary}

In this paper, we have made a number of suggestions regarding syllable structure for an artificial language based on universal principles, that is, based on the types of syllables found in natural language. We suggested in the first part of the paper that it is impractical for syllable structure in an artificial language to be restricted to only simple CV syllables and that some degree of complexity must be allowed. We then made a variety of suggestions as to how that complexity should be restricted by examining each of the units within the syllable. The suggestions are the following: the only type of complex onset that should be allowed is [kw]; the only type of complex nucleus that should be allowed is one with a diphthong [y] off-glide as illustrated by the English word "buy" [bay]; and the only type of coda consonant that should be allowed is a nasal, [m] or [n]. These suggestions can be seen as involving the simplest forms of complexity beyond the CV syllable type and thus should be easily accommodated by an artificial language. Regarding other issues of prosody, it was suggested that an artificial language need not adopt a minimal word requirement. And, with respect to word stress, maintaining stress on the initial syllable of all words would be easiest, though this would require syllables with a complex nucleus to be restricted to the first syllable since there would otherwise be a strong tendency to stress such syllables elsewhere in the word. The suggestions offered here strike a balance between the desire forsimple 
syllable structure with the necessity of having enough syllable types available to express uniquely all the different words in the language.

\section{References}

Blevins, Juliette. 1995. The Syllable in Phonological Theory. In John Goldsmith (ed.), 206-244.

Cresswell, John \& John Hartley. 1968. Esperanto. London: The English Universities Press.

Davis, Stuart. 1988. Topics in Syllable Geometry. New York: Garland.

Davis, Stuart \& Michael Hammond. 1995. On the Status of On-glides in American English. Phonology 12, 159-182.

Goldsmith, John (ed.). 1995. The Handbook of Phonological Theory. Cambridge, MA: Blackwell.

Halle, Morris \& Jean-Roger Vergnaud. 1987. An Essay on Stress. Cambridge, MA: MIT Press.

Hayes, Bruce. 1995. Metrical Stress Theory. Chicago: University of Chicago Press.

Hyman, Larry. 1977. On the Nature of Linguistic Stress. Studiesin Stres sand Accent, Southern California Occasional Papers in Linguistics 4, 37-82.

Kager, Ren. 1999. Optimality Theory. Cambridge: Cambridge UniversityPress.

McCarthy, John \& Alan Prince. 1995. Prosodic Morphology. In John Goldsmith (ed.), 318-366.

Prince, Alan \& Paul Smolensky. 1993. Optimality Theory: Constraint Interaction in Generative Grammar. Boulder: Rutgers University and University of Colorado. 